Occupational Health" carries out active work directed to working conditions optimisation, protecting workers' health, and decrease working losses dealing with occupational and work related diseases, invalidity and premature mortality, that will result in creation of the best conditions to increase the population and working longevity. The plan is focused to ensure full coverage of all workers, including those employed in the informal sector, small and medium enterprises and agriculture, as well as migrant workers, etc. in the field of occupational health.

Conclusions The concept of implementation of state policy aimed at Russian workers' health maintaining for the period up to 2020 and future includes the national action plan on workers' health and contains main principles and measures directed occupational health and safety ensuring.

\section{RELIGIOUS AND CULTURAL FACTORS: BARRIERS TO DEVELOPING INTERVENTIONS AND SAFETY COUNTERMEASURES}

Ahsan Kayani, Mark King, Judy Fleiter. Queensland University of Technology (QUT), Centre for Accident Research and Road Safety - Queensland (CARRS-Q), Australia

\subsection{6/injuryprev-2016-042156.92}

Background Most of the world's people live in developing countries yet there has been limited application of scientific methods of injury control in many of them. Traditional cultural and religious values can act as a barrier to health-promoting and injury prevention behaviours, in general and especially in relation to road safety, and may also contribute to risk-taking behaviours. Such beliefs, including fatalism and superstition, can present significant challenges for health advocates who aim to change behaviour in order to avert road crashes and diminish their consequences.

Methods Qualitative research was undertaken in Islamabad, Rawalpindi and Lahore in Pakistan with a range of drivers, religious orators, police and policy makers to explore cultural and religious beliefs and their association with risky road use, and to understand how they might affect development of road safety interventions.

Results Overall, findings indicated a variety of strongly-held religious and cultural beliefs (such as fatalism and superstition), many that were non-scientific in nature, about road crash causation and ways in which people protect against harm on the road. The findings highlight a range of issues, including the identification of aspects of beliefs that have complex social implications when designing safety intervention strategies. The pervasive nature of such beliefs can affect road user behaviour by reinforcing the presumption that the individual has no part to play in safety, thereby supporting continued risk taking behaviours.

Conclusions The mechanisms of culture and religion should be taken into account when trying to change attitudes and behaviours relating to public health. For effective road safety interventions in developing countries, it is important to understand the prevailing cultural and social beliefs towards road crashes which influence behaviour and thereby preventive measures and responses to interventions adapted from developed countries.

\section{Falls}

Parallel Mon 1.5

\section{OLDER ADULT FALL PREVENTION — GETTING TO OUTCOME MEASURES IN THE CLINICAL SETTING}

${ }^{1}$ Robin Lee, ${ }^{1}$ Erin Parker, ${ }^{1}$ Gwendolyn Bergen, ${ }^{2}$ Colleen Casey, ${ }^{2}$ Elizabeth Eckstrom, ${ }^{3}$ Frank Floyd, ${ }^{1}$ Ann Dellinger, ${ }^{1}$ Grant Baldwin. ${ }^{1}$ Centres for Disease Control and Prevention/ National Centrefor Injury Prevention and Control, USA; 'Division of General Internal Medicine and Geriatrics, Department of Medicine, Oregon Health and Science University, USA; ${ }^{3}$ United Health Services, USA

\subsection{6/injuryprev-2016-042156.93}

Background Worldwide falls are a threat to adults 65 and older. In the United States, one in three older adults will fall annually costing the health care system $\$ 34$ billion. With U.S. fall rates on the rise, and 10,000 older adults turning 65 each day, falls are a major health threat.

Description of the problem Falls can be prevented by addressing modifiable risk factors (e.g., medication usage, vitamin D deficiency, vestibular disorders, vision deficits) with effective clinical interventions; however, few older adults talk to their health care provider about falls. Therefore, CDC launched the STEADI (Stopping Elderly Accidents, Deaths, and Injuries) initiative. STEADI uses established clinical guidelines and evidence-based interventions to empower primary care providers to screen, assess, and treat elderly patients' modifiable fall risk factors. This session describes the STEADI implementation process, key implementation steps, and subsequent health outcomes.

Results STEADI was implemented in multiple health systems. Critical in implementing STEADI was the proactive leadership of clinical champions embedded within the clinical practice; the identification of relevant quality and financial drivers; the modification of electronic health record tools; and the adoption of a STEADI clinical workflow for patients, staff, and providers that aligned with existing workflows. Preliminary measures in one setting indicate providers have screened upwards of 70 per cent of their older adult patients, and hospitalizations and emergency department visits for fall-related injuries are declining.

Conclusion Fall interventions offered in clinical settings can prevent falls among older adults, thereby improving their health, independence, and quality of life. These interventions can reduce medical costs associated with fall injuries, including hospitalisation costs for traumatic brain injuries and hip fractures. Using these data, CDC is disseminating the adoption of STEADI nationwide.

\section{$94 \quad$ FALLS IN MIDDLE-AGED ADULTS PRESENTING TO EMERGENCY DEPARTMENTS IN QUEENSLAND, AUSTRALIA: RISK FACTOR EXPLORATION}

Emily Li, Michele Clark, Kristiann Heesch, Kirsten Vallmuur. Queensland University of Technology, Australia

\subsection{6/injuryprev-2016-042156.94}

Background Falls represent a significant public health issue, with previous studies focusing on older adults. With an ageing population and corresponding escalation of health expenditure, the need to target the current and future health of middle-aged population is evident. However, little is known about the characteristics of fall risk factors in middle-aged populations. This study 